\title{
Multidisciplinary, multisite trauma team training during COVID-19: lessons from the first virtual E-S.T.A.R.T.T. course
}

\author{
Garrett G.R.J. Johnson, MD \\ Jacqueline Beaumont, MD \\ John Damian Paton-Gay, MD \\ Sandy Widder, MD, MHA, MSc \\ Lawrence M. Gillman, MD, \\ MMedEd
}

Accepted September 20, 2021

\author{
Correspondence to: \\ L.M. Gillman \\ Department of Surgery \\ University of Manitoba \\ GF439 - 820 Sherbrook St \\ Winnipeg MB R3A 1R9 \\ Lawrence.Gillman@umanitoba.ca
}

Cite as: Can J Surg 2021 November 10; 64(6). doi: 10.1503/cjs.009921

\section{SUMmaRY}

Trauma care delivery is a complex team-based task that requires deliberate practice. The COVID-19 pandemic has not diminished the importance of excellent trauma team dynamics. However, the pandemic hampers our ability to gather safely and train together. A mitigating solution is the provision of high-fidelity simulation training in a virtual setting. The Simulated Trauma and Resuscitation Team Training (S.T.A.R.T.T.) course has provided multidisciplinary trauma team members with skills in crisis resource management (CRM) for nearly 10 years. It has promoted collaborative learning from coast to coast, as the course typically runs at our national surgical and trauma meetings. In response to COVID-19 challenges, the course content has been modified to virtually connect 2 centres in different provinces simultaneously. High participant satisfaction suggests that the new virtual E-S.T.A.R.T.T course is able to continue to help providers develop important CRM skills in a multidisciplinary setting while remaining compliant with COVID-19 safety precautions.

$\mathbf{T}$ he importance of simulation for trauma education cannot be overstated. Studies have shown that nontechnical skills and overall trauma team performance improves after simulation training. This has important implications for patient outcomes, including improving time to diagnosis and treatment. ${ }^{1}$ Unfortunately, the COVID-19 pandemic has had broad and lasting impact on all aspects of health care delivery, particularly the ability to offer simulation-based learning opportunities to health care professionals. Despite this, the requirement for highly trained and qualified trauma teams has remained unchanged, as traumatic presentations to hospitals in Canada have largely remained at prepandemic levels. ${ }^{2}$ Therefore, there is an ongoing need to provide safe, high-quality team-based trauma training to providers so that they can continue to care for these patients.

There are many challenges to delivering high-quality trauma education during the pandemic. With the competing demands of the pandemic, trauma education has been less of a priority for many institutions. New vaccines have diminished the threat of the virus somewhat; however, physical distancing requirements and travel restrictions have remained. Simulation centres have to consider the optimal use of essential materials and resources, as well as changes in layout for teaching, all while maintaining a semblance of reality. With the rise of variants and predictions that COVID-19 will become endemic, these requirements may be longlasting. ${ }^{3}$ Therefore, providing new simulation modalities is becoming increasingly important. Platforms such as video-led teaching, computer games, virtual patients and other technologies are becoming more common now that centres are attempting to adjust to the new pandemic learning environments. ${ }^{4}$

The Simulated Trauma And Resuscitation Team Training (S.T.A.R.T.T.) course has been providing trauma personnel with multidisciplinary team 
training for nearly 10 years. ${ }^{5}$ The course is taught at national surgical and trauma meetings, and therefore promotes national collaboration and learning opportunities. In this course, surgeons, physicians, registered nurses (RNs), respiratory therapists (RTs) and prehospital personnel come together to learn crisis resource management (CRM) skills in a high-fidelity simulated setting. ${ }^{6}$ This affords participants a relatively safe environment (both psychologically and physically from the threat of patientacquired infection) in which to practise communication, teamwork, leadership, followership, situational awareness and resource utilization skills. The S.T.A.R.T.T. course has relied heavily upon national or international experts travelling between sites to provide in-person instruction. ${ }^{7}$ Both of these factors have required major adjustment during the pandemic to ensure well-being for all and that government-mandated safety restrictions can be followed. In response, we developed the first virtual E-S.T.A.R.T.T. course.

Though the pandemic has been associated with multiple hardships, one of its unexpected benefits has been the relative familiarity that the average person has gained with using virtual teleconferencing software. We took advantage of this factor to allow increased connectedness in our course while keeping people physically separate. We ran 2 courses simultaneously: 1 in Edmonton, Alta., and 1 in Winnipeg, Man. Each site was linked by audiovisual teleconferencing software (Zoom video communications, Inc.) to allow participants to communicate between locations. Each site consisted of a group that was smaller $(<10$ people) than the typical course size. This allowed for physical distancing during the nonclinical components of the course and was compliant with government and organizational restrictions. By connecting electronically, 15 clinicians and 6 instructors/affiliates could participate across both sites. Each site would alternate between observation and participation in simulation trauma scenarios (see course schedule in Appendix 1, available at canjsurg.ca). During the participation phases, teams consisted only of the individuals from their respective site, but afterwards received expert and peer feedback from those present in person as well as from those watching from the alternate site (Figure 1). Participants ranked this process quite highly. All 15 participants agreed to the statements: "Do you feel observing others during their simulations enhanced your learning?" and "Do you feel having other centres involved in the course enhanced your learning?" in the post-participation survey. Participants commented that the virtual component allowed them to learn through both observation and participation and allowed a larger number of perspectives to be shared than would be possible at a single location. Previous versions of the S.T.A.R.T.T. course had learners actively participating in all the simulations, as it was felt by the course curriculum committee that hands-on activities would be more highly valued than observation alone. However, participants in this course pointed out that having time to observe others gave them time to rest mentally and to recover from the challenging scenarios and gave them a unique "bird's eye view" from which to pick up on subtleties that they may have otherwise missed when immersed in the scenarios. It also gave them a feeling of camaraderie with the participants from the other sites and a boost to their confidence to see that all participants can struggle at times in these difficult situations. This was a valuable lesson and something we plan to incorporate into all courses moving forward.

Didactic course instruction was also performed semivirtually. Two instructors, each with a particular focus of expertise, taught their portion of the course from their respective locations, Winnipeg and Edmonton. Participants from the alternate site watched a portion of the didactic teaching live on a screen but could be seen by the instructor and ask questions in real time.

Another revision to the course curriculum included the provision of an initial "trial run" whereby participants went through their first simulation activity at the beginning of the course before receiving any didactic instruction and having only read the course textbook beforehand. The introduction of this new trial run was rated quite highly by participants. At the end of the day, reflection on the trial run allowed participants to reflect on their improvements throughout the course. Participants also felt that this sequence allowed for immediate immersion in the topic and allowed subsequently more engagement with didactic sessions. Virtual instruction in particular can be difficult to engage with for long periods of time,${ }^{8}$ so this trial run may have helped to alleviate some of that burden. Audience participation and Socratic methods were also used to engage the audience during virtual teaching. Alternating instructors between sites allowed participants breaks from watching the screen.

A final adjustment to the course was the introduction of a COVID-19 trauma patient scenario. Enhanced personal protective equipment (PPE) added a highly pertinent area of complexity for participants to practise and balance against their CRM skills. Participants had to don and doff appropriate PPE for aerosol-generating procedures. Physical barriers provided to limit the spread of aerosols could impair verbal communication. Surgical masks and eye protection were worn throughout the entire course (Figure 2).

As in our real work lives during the pandemic, there were some challenges and some areas for future course improvements. We specifically sought out feedback on the quality of the audiovisual materials and the virtual content of the course. The microphone input was 


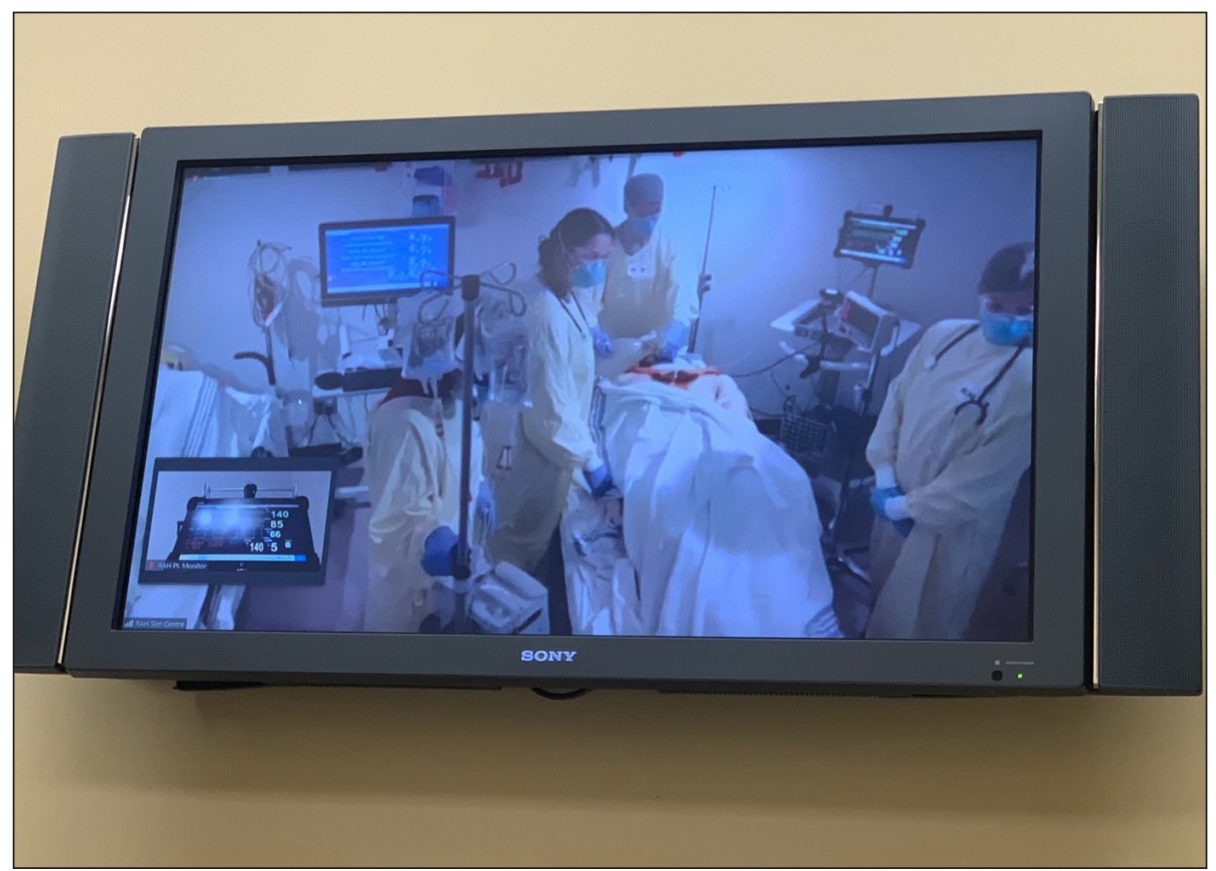

Fig. 1. Participants in Manitoba remotely observe the team in Edmonton completing a simulation scenario. Vital signs are displayed in the lower left corner.

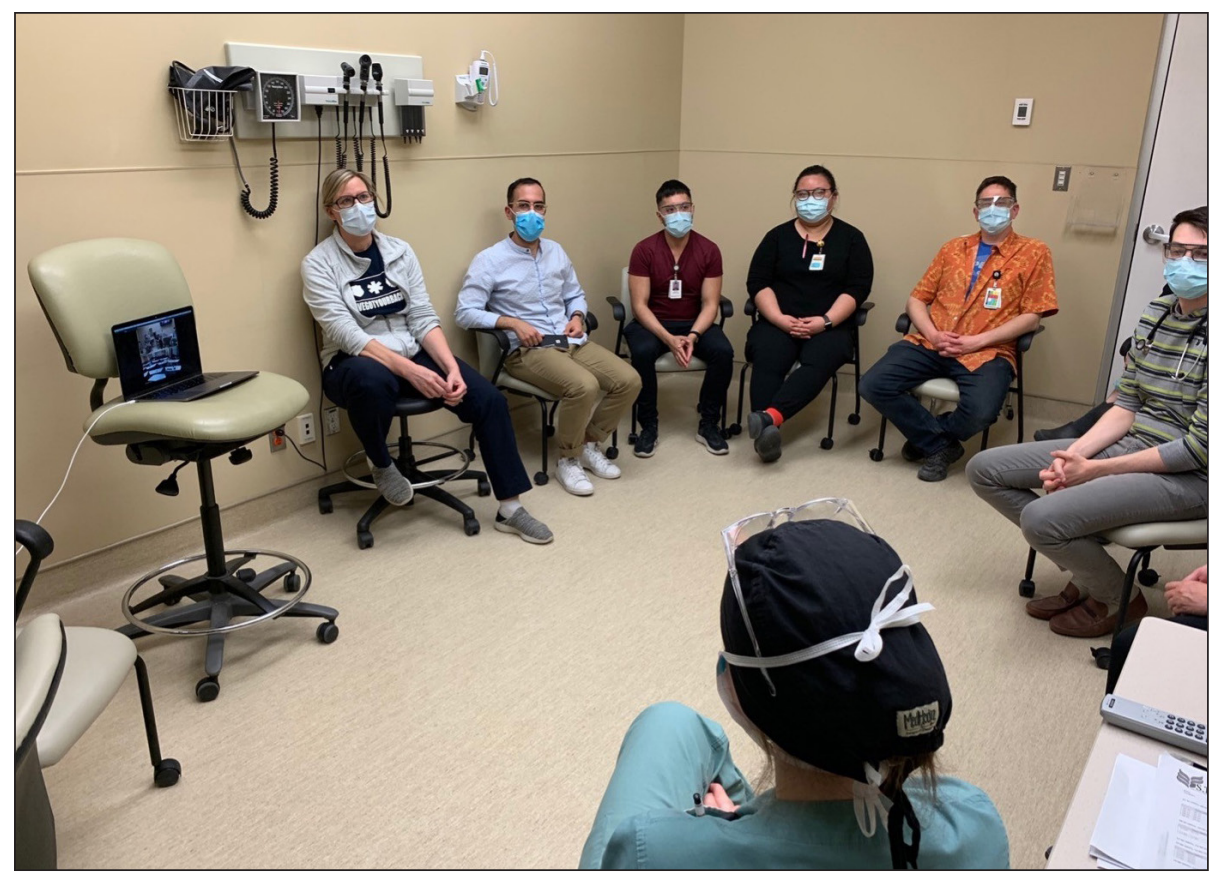

Fig. 2. Participants in Edmonton are given a virtual seat in the "circle of safety" and participate in the debrief of the scenario in Winnipeg.

sometimes unreliable; participants in Winnipeg occasionally had a difficult time hearing the Edmonton participant conversations. This could be addressed in future courses with multiple microphones to enhance audio uptake. Despite this limitation, participants at both sites felt that the observation portion was valuable and should be maintained in future iterations of the course. Furthermore, as always, overall ratings of the course were overwhelmingly positive (Figure 3 ). Participants stated they would be willing to register for a virtually connected course in the future, even if the threat of COVID-19 were eliminated. Time zones were another consideration. Edmonton and Winnipeg are separated by only 1 hour; however, the full-day course had to be adjusted to fit within both simulation facilities' 8 -hour workdays. For future courses, as 


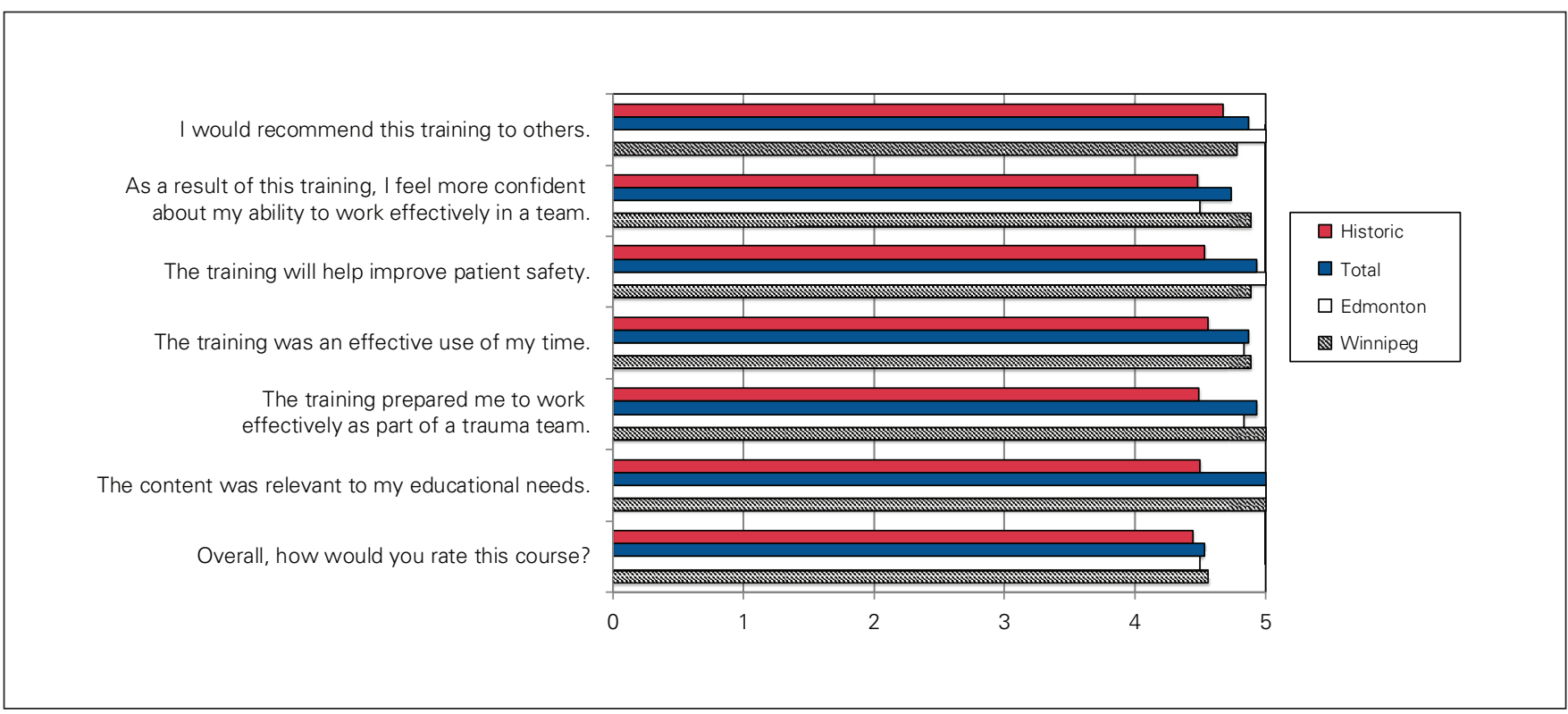

Fig. 3. Participant responses to A) satisfaction survey and B) quality of virtual components. Responses are stratified by participant location and compared with historical data from previous courses. Responses were rated on a 5-point Likert scale.

geographic locations become more disparate, scheduling at a convenient time (i.e., during daytime hours) will be difficult.

\section{Conclusion}

Overall, the first virtual E-S.T.A.R.T.T. course was a success. Participants were able to develop important CRM skills in a multidisciplinary setting while remaining compliant with COVID-19 safety restrictions. Audiovisual technology used in our everyday clinical and administrative lives allowed us to link providers from multiple physical locations in order to share expertise and perspectives. All of this was done without the potential disadvantages of physical travel such as work absence, financial costs, and possible disease transmission. Despite some minor technical difficulties, participants ranked the course quite highly, and valued the virtual observation components. Semivirtual trauma simulation training, when optimized, offers a new method of instruction with multiple benefits. Over time, as the threat of COVID-19 diminishes, we expect many of these advantages to remain. E-S.T.A.R.T.T. may be an opportunity to connect and train diverse trauma providers for the foreseeable future.

Affiliations: From the Department of Surgery, University of Manitoba, Winnipeg, Man. (Johnson, Beaumont, Gilman); and the Department of Surgery, University of Alberta, Edmonton, Alta. (Paton-Gay, Widder).

Competing interests: None declared.

Contributors: All authors contributed substantially to the conception, writing and revision of this article and approved the final version for publication.
Content licence: This is an Open Access article distributed in accordance with the terms of the Creative Commons Attribution (CC BY-NC-ND 4.0) licence, which permits use, distribution and reproduction in any medium, provided that the original publication is properly cited, the use is noncommercial (i.e., research or educational use), and no modifications or adaptations are made. See: https://creativecommons.org/licenses/by-nc-nd/4.0/

\section{References}

1. Barleycorn D, Lee GA. How effective is trauma simulation as an educational process for healthcare providers within the trauma networks? A systematic review. Int Emerg Nurs 2018;40:37-45.

2. Ball CG. The Canadian-specific impact of COVID-19 on severe injuries from intentional violence, unintentional trauma and suicide-related causes. Can 7 Surg 2021;64:E228-9.

3. Phillips $\mathrm{N}$. The coronavirus is here to stay - here's what that means. Nature 2021;590:382-4.

4. Alves Bastos E, Castro M, Lucchetti G. Simulation in healthcare education during and after the COVID-19 pandemic. Simul Healthc 2020;15:298-9.

5. Ziesmann MT, Widder S, Park J, et al. S.T.A.R.T.T.: development of a national, multidisciplinary trauma crisis resource management curriculum-results from the pilot course. 7 Trauma Acute Care Surg 2013;75:753-8.

6. Johnson GG, Brindley PG, Gillman LM. Fidelity in surgical simulation: further lessons from the S.T.A.R.T.T. course. Can 7 Surg 2020;63:E161-3.

7. Gillman LM, Widder S, Clément J, et al. Trauma simulation in bilingual Canada: Insurmountable barrier or unexpected strength? Insights from the first bilingual S.T.A.R.T.T. course. Can 7 Surg 2016;59:80-2.

8. Wilcha R-J. Effectiveness of virtual medical teaching during the COVID-19 crisis: systematic review. FMIR Med Educ 2020;6:e20963. 